\title{
TUBERCULOSE ENDOBRÔNQUICA: SÉRIE DE CASOS
}

\author{
ENDOBRONCHIAL TUBERCULOSIS: CASE SERIES
}

Eduardo Felipe Barbosa Silva

DOI - 10.5935/2236-5117.2020v57a07

\section{RESUMO}

Objetivos. Descrever as características clínicas, radiológicas, microbiológicas e endoscópicas de nove pacientes com tuberculose endobrônquica.

Métodos: Estudo descritivo e retrospectivo, baseado em prontuários de pacientes com tuberculose endobrônquica no período de junho de 2014 a fevereiro de 2016. Sexo, idade, quadro clínico, achados broncoscópicos, microbiologia, anatomopatologia e radiologia foram avaliados.

Resultados: Nove pacientes foram avaliados. 0 sexo feminino predominou com $66,6 \%$ dos casos $(6 / 9)$ e a idade média foi $40,8 \pm 17,5$ anos. Tosse foi a principal queixa $(8 / 9,88,9 \%)$. Cinco pacientes tinham hipóteses diagnósticas clínicas iniciais de pneumonia ou neoplasia $(55,6 \%)$. A radiografia de tórax sugeria o diagnóstico de tuberculose pulmonar em seis casos $(6 / 9,66,7 \%)$, mas nenhum aventou a hipótese de tuberculose endobrônquica. A lesão endobrônquica mais observada foi a caseosa ativa $(4 / 9,44,4 \%)$. A pesquisa de bacilos álcool-ácido resistentes (BAAR) no escarro foi negativa em cinco pacientes, e, no lavado brônquico, foi positiva em três pacientes (3/9, 33,3\%). A cultura do lavado brônquico obteve crescimento de Mycobacterium tuberculosis em cinco pacientes (5/7, $71,4 \%)$. Não foi observada resistência do à rifampicina ou isoniazida em todas amostras testadas $(7 / 7,100 \%)$. As biópsias das lesões endobrônquicas demonstraram processo inflamatório crônico granulomatoso em todos pacientes, $(9 / 9,100,0 \%)$.

Conclusões: As características clínicas, radiológicas, microbiológicas e broncoscópicas desta série estão de acordo com a literatura médica. A broncoscopia tem papel fundamental no diagnóstico da TBE através da visualização de lesões endobrônquicas e obtenção de espécimes para exames complementares.

Palavras-Chave. Tuberculose; tuberculose endobrônquica; broncoscopia; diagnóstico
Eduardo Felipe Barbosa Silva - Silva EFB. Médico. Unidade de Broncoesofagologia, Hospital de Base do Distrito Federal, Brasilia, Distrito Federal, Brasil.

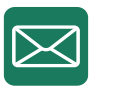

Correspondência: Eduardo Felipe Barbosa Silva. SQSW 306 Bloco B Apartamento 306, Setor Sudoeste, Brasília, Distrito Federal, Brasil. CEP 70673432.

Internet: efbsbpt@gmail.com

Conflito de interesses: os autores declaram não haver conflito de interesses.

\section{ABSTRACT}

Objective: The study aims to describe the clinical, radiological, microbiological, and endoscopic characteristics of nine patients with endobronchial tuberculosis.

Methods: Descriptive and retrospective study based on medical records of patients with endobronchial tuberculosis from June 2014 to February 2016. Sex, age, clinical status, bronchoscopic findings, microbiology, anatomopathology, and radiology were imposed.

Results: Nine patients were subject to fees. The female gender predominated, $66.6 \%$ of the cases $(6 / 9$,$) and the age$ was $40.8 \pm 17.5$ years. The cough was the main symptom (8/9, 88.9\%). Five patients had initial clinical diagnostic hypotheses of pneumonia or neoplasia $(55.6 \%)$. A chest $X$-ray suggests pulmonary tuberculosis diagnosis in six cases $(6 / 9,66.7 \%)$, but there is still no chance of endobronchial tuberculosis. The most observed endobronchial lesion was the active caseous lesion (4/9, 44.4\%). A sputum alcohol-resistant bacilli (BAAR) test was negative in five patients, and, in bronchial lavage, it was positive in three patients (33.3\%). The culture of bronchial lavage grows Mycobacterium tuberculosis in five patients $(71.4 \%)$. No resistance to rifampicin or isoniazid was observed in all tested samples $(7 / 7,100 \%)$. The biopsies of the endobronchial lesions demonstrated the chronic granulomatous inflammatory process in all patients. 
Conclusions: The clinical, radiological, microbiological, and bronchoscopic characteristics of this series follow the medical literature. Bronchoscopy has a fundamental role in diagnosing endobronchial tuberculosis through responses to endobronchial lesions and sample exams for complementary exams.

Keywords: Tuberculosis; endobronchial tuberculosis; Bronchoscopy; diagnosis.

\section{INTRODUÇÃO}

A tuberculose endobrônquica consiste na infecção tuberculosa da árvore traqueobrônquica, com ou sem o envolvimento do parênquima pulmonar. ${ }^{1,2}$ Apesar de sua importância, a doença permanece subdiagnosticada. ${ }^{2-4}$ Estudo prévio, no qual todos pacientes com tuberculose pulmonar ativa foram submetidos à broncoscopia, observou tuberculose endobrônquica em $54,3 \%$ dos casos. ${ }^{5}$ Acredita-se que a implantação direta do bacilo da tuberculose oriundo da lesão parenquimatosa no brônquio, a infiltração ou erosão com protrusão luminal de linfonodos mediastinais e a disseminação hematogênica e/ou linfática originem essa forma de tuberculose. ${ }^{2,3}$

O padrão ouro para o diagnóstico da tuberculose endobrônquica consiste na visualização por broncoscopia de lesões endobrônquicas associado à confirmação histológica e/ou microbiológica de amostras coletadas por endoscopia. ${ }^{3,4,6}$ As lesões endobrônquicas podem ser classificadas conforme Chung-Lee em: caseosa ativa, granular, tumoral, fibroestenótica, bronquite inespecífica, edematosahiperêmica e ulcerativa. ${ }^{3}$

O tratamento consiste na combinação de drogas tuberculostáticas de primeira linha (rifampicina, isoniazida, pirazinamida e etambutol), conforme recomendações vigentes. ${ }^{7}$ Apesar do efetivo tratamento medicamentoso, o curso e o prognóstico da tuberculose endobrônquica são variáveis, indo desde a completa resolução da doença até severa complicação, como broncoestenose. ${ }^{2-4}$

Dessa forma, o objetivo desse estudo é descrever as características clínicas, radiológicas, microbiológicas e endoscópicas de nove pacientes com tuberculose endobrônquica.

\section{MÉTODOS}

Foram retrospectivamente revisados os prontuários de nove pacientes com o diagnóstico de tuberculose endobrônquica atendidos no período de junho de 2014 a fevereiro de 2016. Características como sexo, idade, quadro clínico, achados broncoscópicos, microbiologia, anatomopatologia e radiologia foram avaliados.

O estudo foi aprovado pelo Comitê de Ética e Pesquisa da Fundação de Ensino e Pesquisa em Ciências da Saúde (CAAE: 63592116.8.0000.5553).

\section{RESULTADOS}

Dos nove pacientes avaliados, houve predomínio do sexo feminino com 6 casos $(66,6 \%)$. A média de idade foi de $40,8 \pm 17,5$ anos. Tosse foi a queixa mais relatada, estando presente em oito casos $(8 / 9,88,9 \%)$. Outras queixas foram dor torácica $(5 / 9,55,6 \%)$, emagrecimento $(5 / 9,55,6 \%)$, febre $(2 / 9,22,2 \%)$, sudorese noturna $(2 / 9,22,2 \%)$ e dispneia $(2 / 9,22,2 \%)$. Dos seis pacientes que realizaram sorologia para vírus da imunodeficiência humana (HIV), uma paciente apresentava síndrome da imunodeficiência adquirida (SIDA) $(1 / 6,16,7 \%)$, em três casos não foi solicitada a sorologia. Em cinco pacientes, as hipóteses diagnósticas clínicas iniciais foram de pneumonia ou neoplasia $(55,6 \%)$. Seis pacientes possuíam achados radiológicos sugestivos de tuberculose pulmonar conforme laudos radiológicos $(6 / 9,66,7 \%)$, mas em nenhum a hipótese de tuberculose endobrônquica foi aventada, apesar da presença de atelectasia em três casos $(3 / 9,33,3 \%)$ e estenose brônquica em dois pacientes $(2 / 9,22,2 \%)$, (tabela 1 e figura 1$)$. 
Tabela 1. Características clínicas, radiológicas, microbiológicas e endoscópicas dos pacientes avaliados.

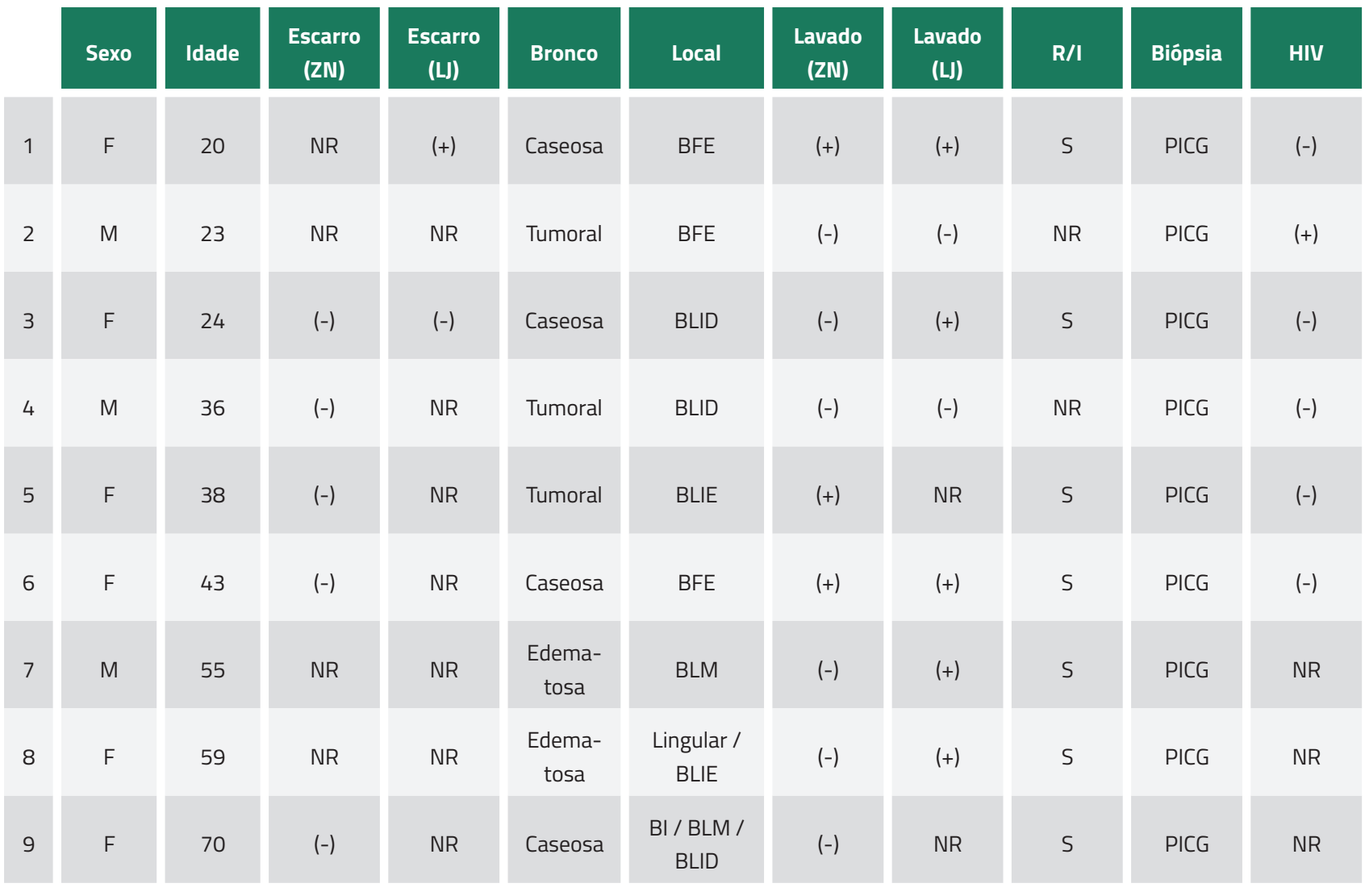

M: masculino; F: feminino; NR: não realizado; Escarro (ZN): pesquisa de bacilo álcool-ácido resistente no escarro pela técnica de Zielh-Neelsen; Escarro (LJ): cultura do escarro em meio de Lowestein-Jensen; Lavado (ZN): pesquisa de bacilo álcool-ácido resistente no lavado brônquico pela técnica de Zielh-Neelsen; Lavado (LJ): Cultura do lavado brônquico em meio de Lowestein-Jensen; Bronco: lesão endoscópica conforme classificação de Chung-Lee; S: sensível; PICG: processo inflamatório crônico granulomatoso; R/I: Sensibilidade do Micobacterium Tuberculosis à rifampicina/isoniazida; HIV: Sorologia para o vírus da imunodeficiência humana; Local: local da lesão endoscópica na árvore traqueobrônquica; BFE: brônquio fonte esquerdo; BLID: brônquio do lobo inferior direito; BLIE: brônquio do lobo inferior esquerdo; BLM: brônquio do lobo médio; BI: brônquio intermediário.

A pesquisa de bacilos álcool-ácido resistentes (BAAR) no escarro, pela técnica de Ziehl-Neelsen, foi negativa em todos pacientes submetidos a este exame $(5 / 5,100 \%)$, sendo que quatro pacientes não realizaram pesquisa de BAAR no escarro devido a não ter secreção para coleta do exame (tosse seca) ou não solicitação médica. Dois pacientes tiveram amostra de escarro cultivada em meio de Lowestein-Jensen, sendo observado crescimento de Micobacterium Tuberculosis em um paciente (50\%), sete pacientes não realizaram esse método por razões semelhantes às citadas anteriormente (tabela 1).

Na broncoscopia, conforme a classificação de Chung-Lee3, a lesão endobrônquica mais visualizada foi a caseosa ativa, presente em quatro pacientes $(4 / 9,44,4 \%)$. Em três pacientes, foi observado o subtipo tumoral $(33,3 \%)$ e em dois o subtipo edematoso-hiperêmico (22,2\%) (figura 1 ). Os locais mais acometidos pelas lesões endobrônquicas foram o brônquio fonte esquerdo em três pacientes $(33,3 \%)$ e o brônquio do lobo inferior direito em três pacientes (33,3\%). A pesquisa de BAAR, pela técnica de Ziehl-Neelsen, no lavado brônquico foi positiva em três pacientes $(3 / 9,33,3 \%)$. A cultura do lavado brônquico, em meio de Lowestein-Jensen, apresentou crescimento de Micobacterium tuberculosis em cinco pacientes $(5 / 7,71,4 \%)$, dois casos não realizaram esse método diagnóstico, sendo observada sensibilidade à rifampicina e isoniazida em todos pacientes $(7 / 7,100 \%)$. As biópsias das lesões endobrônquicas demonstraram processo inflamatório crônico granulomatoso em todos os pacientes $(9 / 9,100 \%$ ) (tabela 1). 


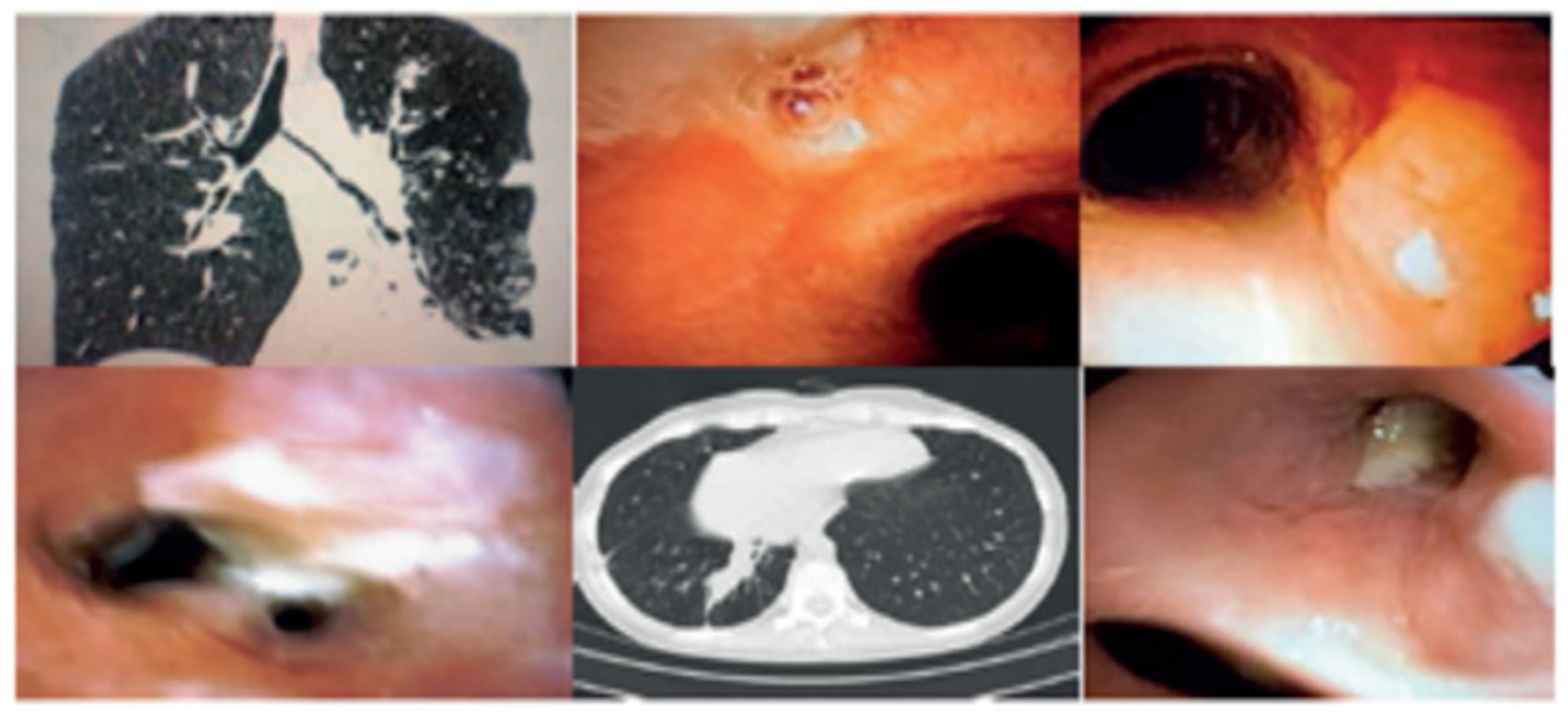

Figura 1. A) Tomografia computadorizada (corte coronal) demonstrando estenose de brônquio fonte esquerdo e redução volumétrica do pulmão esquerdo associado a sinais radiológicos de tuberculose pulmonar ativa em parênquima (paciente 1), B) redução da luz do brônquio fonte esquerdo associada a mucosa brônquica edemaciada, hiperemiada e recoberta com material caseoso de coloração amarelada (paciente 1), C) lesão tumoral em parede medial do brônquio fonte esquerdo (paciente 2, D) mucosa do brônquio do lobo inferior direito edemaciada, hiperemiada e recoberta com material caseoso de coloração amarelada em brônquio do lobo inferior direito (paciente 3), E) tomografia computadorizada com opacidade com componente atelectásico em lobo inferior direito associado a sinais radiológicos de tuberculose pulmonar ativa em parênquima (paciente 3), e F) lesão tumoral vegetante de coloração amarelada em brônquio do lobo inferior direito (paciente 4).

Todos pacientes foram submetidos ao tratamento com tuberculostáticos. Dos seis pacientes que havia informações a respeito da evolução clínica, todos apresentaram melhora clínica $(6 / 6,100,0 \%)$. Nenhum paciente recebeu corticoterapia. Dois pacientes realizaram acompanhamento broncoscópico da tuberculose endobrônquica, porém uma paciente de forma inadequada, pois realizou o exame endoscópico somente no primeiro mês de drogas tuberculostáticas, não sendo possível avaliar a evolução da lesão endobrônquica, enquanto o segundo paciente realizou acompanhamento boncoscópico adequado, sendo observada resolução da lesão endobrônquica, sem estenose ao término do tratamento.

\section{DISCUSSÃO}

A tuberculose endobrônquica acomete preferencialmente indivíduos jovens e do sexo feminino..$^{2-4,6,8} \mathrm{~A}$ razão para o maior comprometimento do sexo feminino parece ser a não expectoração por razões socioculturais, ocasionando maior exposição da árvore traqueobrônquica ao bacilo da tuberculose e posteriormente sua implantação. ${ }^{3-5}$

O quadro clínico é inespecífico, sendo tosse a queixa mais comum. ${ }^{2,4,6,8}$ Febre, anorexia, emagrecimento, expectoração de secreção ou cartilagens, hemoptise e dor torácica também são descritos. Dispneia, estridor e sibilos podem ocorrem na presença de obstrução traqueobrônquica. ${ }^{9,10}$ É frequente a tuberculose endobrônquica mimetizar outras doenças, como asma brônquica e neoplasia11-13. A lesão endobrônquica mais observada no exame broncoscópico é a caseosa ativa, conforme a classificação de Chung-Lee. . $^{2,3}$

Devido ao baixo rendimento da pesquisa de BAAR, pela técnica de Ziehl-Neelsen, no escarro, que varia de 13,6 a 53,3\% em diferentes estudos, uma baciloscopia do escarro negativa não exclui o diagnóstico de tuberculose endobrônquica. 0 baixo 
rendimento é atribuído ao aprisionamento do muco na árvore traqueobrônquica, causado por tecido de granulação. $6,8,14,15$ A pesquisa de BAAR, pela técnica de Ziehl-Neelsen, no lavado brônquico obteve rendimento de $26 \%$, enquanto a cultura, em meio Lowestein Jensen, obteve rendimento de $39,1 \%$ em estudo prévio. ${ }^{15}$ Fato importante a ser relatado é que conforme o tipo de lesão endobrônquica existem variações nos rendimentos do lavado broncoalveolar e das biópsias, sendo que as lesões granularesulcerativas e fibroestenóticas apresentam o melhor e o pior rendimento, respectivamente..$^{15,16}$

A radiografia de tórax é normal em $10 \%$ dos casos de tuberculose endobrônquica. 2,8,12,17 A tomografia computadorizada de alta resolução é superior a radiografia do tórax no diagnóstico da tuberculose endobrônquica. ${ }^{18,19}$ As alterações radiológicas, quando presentes, são semelhantes as observadas na tuberculose pulmonar parenquimatosa ativa, associada a achados que podem sugerir tuberculose endobrônquica, como massas endobrônquicas e broncoestenose. ${ }^{18}$

Apesar do maior comprometimento linfonodal hilar e mediastinal na tuberculose pulmonar em pacientes com síndrome da imunodeficiência adquirida (SIDA), o que predisporia a maior ocorrência de tuberculose endobrônquica, há poucos relatos na literatura dessa associação. As características clínicas, endoscópicas e microbiológicas de pacientes com SIDA são semelhantes aos do que não possuem essa sindrome. ${ }^{20-22}$

Mesmo com o tratamento efetivo com drogas tuberculostáticas, complicações, como estenose brônquica, podem ocorrer. ${ }^{12}$ Idade maior que 45 anos, intervalo maior que três meses entre o início dos sintomas e o tratamento, e os subtipos caseosa ativa, edematoso-hiperêmico, fibroestenótica e tumoral são descritos como fatores predisponentes para complicação. , $3^{3}$

Corticosteroides são prescritos empiricamente na tuberculose endobrônquica, porém seu efeito é controverso. Alguns autores sugerem benefícios dessa modalidade de tratamento, como redução de estenose brônquica e de reações de hipersensibilidade causada pelos tuberculostáticos. ${ }^{23}$ Porém, há estudos que não mostraram benefício do uso de corticosteroides para evitar estenose brônquica. ${ }^{24,25,26}$ A broncoscopia intervencionista (broncoplastia por balão, eletrocautério, laser e stents) e cirurgias são utilizadas nesses pacientes que apresentam evolução desfavorável com estenose. ${ }^{27-29} 0$ acompanhamento da resposta terapêutica e a detecção de complicações devem ser realizados por broncoscopia ou tomografia computadorizada de tórax durante o tratamento com tuberculostáticos, principalmente nos pacientes mais propensos a complicações. 3,10,21,25,26,30

Essa série de casos possui limitações causadas por ser um estudo retrospectivo e pelo não acesso a informações colhidas diretamente com os pacientes.

\section{CONCLUSÃO}

Conclui-se que as características clínicas, radiológicas, microbiológicas e broncoscópicas dessa série de casos estão de acordo com a literatura médica. A broncoscopia tem papel fundamental no diagnóstico da tuberculose endobrônquica com a visualização das lesões endobrônquicas e obtenção de espécimes para exames complementares.

\section{REFERÊNCIAS}

1. Campos J, Ernst G, Borsini E, Garcia A, Blasco M, Bosio M, Salvado A. Tracheobronchial tuberculosis without lung involvement. J Clin Med Res. 2015;7(8):646-8.

2. Sahin F,Yildiz P. Characteristics of endobronchial tuberculosis patients with negative sputum acidfast bacillus. J Thorac Dis 2013;5(6):764-70.

3. Chung HS, Lee JH. Bronchoscopic assessment of the evolution of endobronchial tuberculosis. Chest. 2000;117(2):385-92.

4. Cordovilla R, Jimenez-Massa A. Endobronchial Tuberculosis: a case report and review of the literature. J Bronchology Interv Pulmonol 2009;16(2):121-3.

5. Jung SS, Park HS, Kim JO,Kim SY. Incidence and clinical predictors of endobronchial tuberculosis in patients with pulmonary tuberculosis. Respirology 2015; 20(3):488-95.

6. Qingliang $X$, Jianxin $W$. Investigation of endobronchial tuberculosis diagnoses in 22 cases. Eur J Med Res. 2010;15(7):309-13.

7. Conde MB, Melo FA, Marques AM, Cardoso NC, Pinheiro VG, Dalcin PT et al. III Brazilian Thoracic Association Guidelines on tuberculosis. J Bras Pneumol. 2009;35:1018-48.

8. Lee $\mathrm{JH}$, Park SS, Lee $\mathrm{DH}$, Shin $\mathrm{DH}$, Yang SC,Yoo BM. Endobronchial tuberculosis. Clinical and bronchoscopic features in 121 cases. Chest 
1992;102(4):990-4.

9. Park MJ, Woo IS,Son JW,Lee SJ, Kim DG,Mo EK, Lee MG,Hyun IG,Juna KS. Endobronchial tuberculosis with expectoration of the tracheal cartilages. Eur Respir J. 2000;15(4):800-2.

10. Kashyap S, Solanki A. Challenges in endobronchial tuberculosis: from diagnosis to management. Pulm Med. 2014;2014:594806

11. Matthews Jl, Matarese SL, Carpenter JL. Endobronchial tuberculosis simulating lung cancer. Chest. 1984;86(4):642-4.

12. Lee TH, Sin Fai Lam KN. Endobronchial Tuberculosis simulating Bronchial asthma. Singapore Med J. 2004;45(8):390-2.

13. Saygideger $Y$, Oktay B, Sevgi E, Sever O, Firat $H$, Ardiç S. Bilateral multiple tumor-like endobronchial tuberculosis, diagnosed with bronchoscopic examination. Tuberk Toraks. 2011;59(3):266-70.

14. Burke HL, Haponik EF. Endobronchial tuberculosis in the elderly: a case report and review of the literature. J Bronchology Interv Pulmonol. 1997;4(2):132-5.

15. Ozkaya S,Bilgin S, Findik S, Kok HC, Yuksel C, Atici AG. Endobronchial tuberculosis: histopathological subsets and microbiological results. Multidiscip Respir Med. 2012;7(1):34.

16. Hou G, Zhang T, Kang DH, Wang W, Hu XJ, Wang QYet al. Efficay of real-time Polymerase Chain Reaction for rapid diagnosis of endobronchial tuberculosis. Int J Infect Dis. 2014; 27:13-7.

17. Garg T, Gera K, Shah A. Middle lobe syndrome: an extraordinary presentation of endobronchial tuberculosis. Pneumonol Alergol Pol. 2015;83(5):387-91.

18. Kim Y,Lee KS,Yoon JH,Chung MP,Kim H,Kwon $\mathrm{OJ}, \mathrm{Rhee} \mathrm{CH}$, Han YC. Tuberculosis of the trachea and main bronchi: CT findings in 17 patients. AJR Am J Roentgenol. 1997.168(4):1051-6.

19. Yanardag H, Tetikkurt C, Tetikkurts S, Demirci S, Karayel T. Computed Tomography and bronchoscopy in Endobronchial Tuberculosis. Can Respir J. 2003;10(8):445-8.
20. Judson MA, Sahn SA. Endobronchial Lesions in HIVinfected individuals. Chest. 1994;105(5):1314-23.

21. Alame T, Dierckx P, Carlier S, Sergysels R. Lymph-node perforation into the airway in AIDS- associated tuberculosis. Eur Respir J. 1995;8(4):658-60.

22. Wasser LS, Shaw GW, Talavera W. Endobronchial Tuberculosis in the acquired immunofeciciency syndrome. Chest. 1988;94(6):1240-4.

23. Chan HS, Sun A, Hoheisel GB. Endobronchial Tuberculosis- is corticosteroid treatment useful? A report of 8 cases and review of the literature. Postgrad Med J. 1990;66(780):822-6.

24. De S. Effect of antitubercular treatment on tumorous endobronchial tuberculosis.J Bronchology Interv Pulmonol. 2011;18(2):171-5.

25. Park IW, Choi BW, Hue SH. Prospective study of coricosteroid as an adjunct in the treatment of endobronchial tuberculosisin adults. Respirology. 1997;2(4):275-81.

26. Panigrahi MK, Pradhan G, Mishra P, Mohapatra PR. Actively caseating endobronchial tuberculosis sucessfully treated with intermittent chemotherapy without corticosteroid: a report of 2 cases. Adv Respir Med. 2017;85(6):322-7.

27. Ryu YJ, Kim H, Yu CM, Choi JC, Kwon YS, Kwon OJ. Use of silicone stents for the management of post-tuberculosis tracheobronchial stenosis. Eur Respir J. 2006;28(5):1029-35.

28. Low SY, Hsu A, Eng P. Interventional bronchoscopy for tuberculosis tracheobronchial stenosis. Eur Respir J. 2004;24(3):345-7.

29. Ahmed SNM, Korrungruanq P, Saka H, Asai G, Ise $Y$, KitagawaC et al. Balloon dilatation of a case of tuberculous tracheobronchial stenosis during the course of antituberculous treatment. Case Rep Med. 2015;2015:618394.

30. Kreisel D, Arora N, Weisenberg SA, Saqi A, Krupnick AS, Demetriades AM et al. Tuberculosis presenting as an endobronchial mass. J Thorac Cardiovasc Surg. 2007;133(2):582-4. 